\title{
Damage assessment based on general signal correlation. Application for delamination
}

\section{diagnosis in composite structures}

\author{
Irina Trendafilova ${ }^{\mathrm{a}}$, Roberto Palazzetti ${ }^{\mathrm{b}, *}$, Andrea Zucchelli ${ }^{\mathrm{c}}$ \\ ${ }^{a}$ University of Strathclyde, MAE Department, 75 Montrose Street, G1 1XJ Glasgow, UK \\ ${ }^{\mathrm{b}}$ University of Strathclyde, DMEM Department, 75 Montrose Street, G1 1XJ Glasgow, UK \\ ${ }^{c}$ Univerisity of Bologna, DIN Department, viale del Risorgimento 2, 40134 Bologna, Italy \\ *Corresponding author. roberto.palazzetti@ strath.ac.uk. Tel +44 (0) 1415484294
}

\begin{abstract}
This work presents a Vibration-Based Structural Health Monitoring (VSHM) technique which is developed and applied for delamination assessment in composite laminate structures. It suggests the mutual information as a measure for nonlinear signal cross correlation. The mutual information between two signals measured on a vibrating structure is suggested as a damage metric and its application for the purposes of damage assessment is discussed and compared to the application of the traditional linear signal cross-correlation. The cross correlation is capable to detect linear dependence between two signals and thus can be used for diagnosing damage on linearly vibrating structures. On the other hand the mutual information is a nonlinear metric, and it is shown that it can detect linear as well as nonlinear signal dependence and thus it is particularly appropriate for structures with nonlinear dynamic behaviour and for composite structures as such. The application of the mutual information as a damage metric is demonstrated and discussed first for the case of a simple 2 DOF system with a nonlinear stiffness. Eventually the application of the suggested damage metric is developed and demonstrated for the purposes of delamination diagnosis in a composite laminate beam.
\end{abstract}




\section{Introduction.}

Structures made of composite materials have an increasing importance in many contemporary industrial, civil and military applications and in particular in the aviation field. They are progressively replacing traditional materials due to their better strength and weight, than traditional materials. Composite laminates are probably the widest used composite material, and besides the number of excellent properties laminates present some difficulties, particularly related to their layered nature, which induces the formation of new failure modes. Delamination is probably the most common failure mechanisms for composite materials and it is particularly dangerous because delaminated structures can lose up to $60 \%$ of their initial stiffness, and still remain visibly unchanged.

This work focuses on the use of the vibration response of structures made of composite laminate materials for their integrity and health assessment.

Maintenance and operation costs are usually among the largest expenditures for most structures: an ageing structure may reduce profits with increased maintenance costs and down time and it can become a hazard for its users. The ability to access the integrity of a structure and discover a fault at a rather early stage can significantly reduce these costs. A large class of Structural Health Monitoring (SHM) methods are vibration-based methods, where the state of the structure is assessed using its vibration response (Yang et al., 2007).

Laminates are very difficult to inspect and almost impossible to repair, thus the evaluation of the health state of such structures is a must for most industrial applications. Vibration-Based Structural Health Monitoring (VSHM) methods are becoming increasingly important for composite and composite laminate structures. VSHM methods can be largely divided into two main categories (Yu and Yang, 2007; Yang et al., 2009): model-and non-model based. The first category uses the vibratory model of the structure in order to assess its health and condition, while the latter does not assume and/or require any modeling. Most of the model-based methods 
use a linear structural model. The methods used for structures made of composites tend to be nonmodel based, because of the complexity of material properties which are difficult to model accurately.

Plenty of VSHM methods targeted for structures made of composites use the structural resonant frequencies as damage/delamination features. Doebling et al. (1998) mention that the presence of delamination in a structure would decrease structure's natural frequencies and increase its modal damping as compared to the intact structures. Adams et al. (1975) tested glassreinforced plates to attempt to detect damage after both static and fatigue torsional loading. They found damping to be more sensitive than frequencies for detecting the onset of delamination. Cawley and Adams (1979) apply a frequency-shift-based damage detection routine to several damage cases (holes, saw cuts, crushing with a ball bearing, local heating with a flame, and impact) in composite materials (CFRP plates and honeycomb panels with CFRP faces). They were able to locate low levels of damage accurately. Sanders, et al. (1992) measured the modal parameters on damaged graphite/epoxy beams. Damage was induced by tensile loading the beams to $60 \%, 75 \%$, and $85 \%$ of the ultimate tensile strength. It was diagnosed using a sensitivity method based on the measured natural frequencies. Results agreed well with independently obtained findings based on static stiffness measurements and crack densities from edge replication. Because this damage was approximately uniform throughout the beam, the ability of the method to localize damage was not demonstrated. Diaz Valdes and Soutis (1999) used a novel method known as resonant ultrasound spectroscopy to determine the modal frequencies of a prepreg carbon/epoxy composite laminate beam. They used commercial, brass backed, piezoceramic transducer and a piezoelectric film element bonded near the beam's fixed end and operated as actuator and sensor respectively. Changes of the modal frequencies after delamination initiation, compared to those of a non-delaminated specimen, gave a good indication of the degree of damage, demonstrating the feasibility of using measured changes in 
the vibration characteristics to detect damage. In Minak et al. (2010) the authors make use of the resonant frequencies of a composite beam and develop a pattern recognition procedure for the purposes of delamination diagnosis.

But it should be noted that there are a number of examples when these frequencies turn out to be insensitive to a certain kind of damage especially in its initial state when it has not developed enough (Yang et al., 2009; Nichols et al., 2004). It should be also noted that structures made of composites on a lot of occasions demonstrate quite well expressed nonlinear behaviour, while most of the above mentioned methods use a linear model. Traditional spectrum analysis and modal analysis are applicable to structures with linear dynamic behaviour and thus strictly speaking they cannot be applied to structures made of composites. Moreover on a lot of occasions the measured vibration response signal from structures made of composites is a nonlinear one and thus it is difficult and on some occasions even impossible to extract information, including the natural frequencies, from its frequency domain representation. Thus most of the above mentioned methods might be inapplicable for structures made of composites.

Monitoring methods based on the time-domain vibration signatures represent a relatively new paradigm in SHM (Nichols et al., 2004; Trendafilova, 2006; Trendafilova et al., 2008). These methods are mostly based on non-linear dynamics tools and signal analysis and most of them utilize statistical characteristics. They represent a very attractive alternative, especially for structures made of composites, since they do not assume any model or linearity of the structure under interrogation and they only require the measured structural vibration signals in the current and possibly in a baseline (undamaged) state. The signal cross-correlation was considered for the purposes of damage assessment in (Wang et al., 2010) in a different context where the authors suggest a vector damage measure. The application suggested here is much simpler and straightforward to apply, which will enhance the practical application of the method. The development here is in the extension of the idea of cross correlation for nonlinear signals and for 
nonlinear signal dependence. The information and the entropy of vibrating structures were first considered by Nichols (2006) and their application for nonlinearity detection purposes was suggested by Overbey et al. (2009). In (Trendafilova et al., 2012) the authors of the present paper consider the application of cross correlation and the mutual information for damage and delamination detection in freely vibrating structures. This paper extends and enhances the application of the mutual information to real structures and especially to composite structures subjected to unknown random excitation. It introduces a simple damage index, which is capable of detecting the presence and the extent of damage and locating it within the structure. The method is further demonstrated on a composite beam, for which it is proven to detect and localize different delamination sizes and scenarios. The study also offers a comparison between the performance of the cross-correlation and the mutual information for cases of detecting linear and nonlinear damage in a simple simulated 2 DOF example. A similar comparison is provided for the case of delamination diagnosis in a composite beam, where the mutual information is shown to have superior performance.

The rest of the paper is organized as follows. The concepts of cross-correlation and mutual information between two signals are introduced in the context of their application for structural damage detection in $\& 2 . \& 3$ considers the 2 DOF system example and $\& 4$ is dedicated to application of the suggested metrics for delamination detection in a composite beam. Eventually some results are introduced and discussed (\&5), and the paper is concluded with a discussion (\&6). 


\section{Background of the method.}

\subsection{Signal cross correlation and its application as a damage metric.}

Cross-correlation is a measure of similarity of two signals as a function of a time-lag applied to one of them. If $x_{i}(t)$ and $x_{j}(t)$ are two signals, their cross correlation is defined as follows (Bendat and Piersol 2011):

$R_{x_{i} x_{j}}(\tau)=\lim _{T \rightarrow \infty} \frac{1}{T} \int_{0}^{T}\left[x_{i}(t)-\mu_{i}\right] \cdot\left[x_{j}(t+\tau)-\mu_{j}\right] d t$

where $\mu_{i}$ and $\mu_{j}$ are the mean values of $x_{i}(t)$ and $x_{j}(t)$ respectively. Or for discrete signals:

$$
R_{x_{i} x_{j}}(m)=\lim _{N \rightarrow \infty} \frac{1}{N} \sum_{n=1}^{N}\left[x_{i}(n)-\mu_{i}\right] .\left[x_{j}(n+m)-\mu_{j}\right]
$$

The cross correlation is a signal as well. It has a maximum when the two signals are aligned. The normalized cross-correlation between two signals is defined as (Bendat and Piersol 2011):

$\rho_{x_{i} x_{j}}(m)=\frac{R_{x_{i} x_{j}}(m)}{\sqrt{R_{x_{i} x_{i}}(0) \cdot R_{x_{j} x_{j}}(0)}}$

Where $R_{x_{i} x_{i}}$ and $R_{x_{j} x_{j}}$ are the autocorrelations of $x_{i}$ and $x_{j}$ respectively. It should be noted that $\left|\rho_{x_{i} x_{j}}(m)\right| \leq 1$ for all $m$.

If $x_{j}$ is the same signal as $x_{i}$ their cross-correlation will have a maximum for $m=0$. If $x_{i}$ and $x_{j}$ are linearly related $\left(x_{j}\right.$ is a shifted and amplified/attenuated version of $\left.x_{i}\right)$, then their cross-correlation will have a maximum (and their normalized cross-correlation will be 1) for the shift between the two signals.

It was shown in Wang et al. (2010) Yang et al. (2009) that the cross correlation between two signals measured on a vibrating structure for the case of random white noise excitation depends on the structural characteristics only. The cross correlation measures linear dependence between two signals. For a linearly vibrating structure (or one with close to linear behaviour) the two 
signals $x_{i}$ and $x_{j}$ will be linearly related and their maximum normalized cross correlation will be close to 1 .

The introduction of damage changes the physical properties of the system and so do characteristics like mass, stiffness and hence modal parameters. So the cross-correlation between the two measured signals will change at the introduction of damage. In general it will go down since the dependence between the two signals at the introduction of damage will decrease. Thus it is argued here that the cross correlation between two signals measured on a vibrating structure for the case or random white noise excitation can be used for damage assessment. And accordingly the maximum normalized cross correlation is suggested as a damage metric:

$\Omega_{x_{i} x_{j}}=\max _{m} \rho_{x_{i} x_{j}}(m)$

\subsection{The mutual information and its application as a damage metric.}

A lot of vibrating systems cannot be considered linear especially at high amplitude vibrations, and for such systems a nonlinear analogue of the cross-correlation, the mutual information, is suggested here. The mutual information is a theoretic idea that connects two signals and it determines the amount of information that one of the signals "learns" from the other, or in other words, it determines their mutual dependence in terms of information (Trendafilova et al., 2001; Kantz and Schreiber, 2004; Trendafilova, 2006; Roshni et al., 2008; Trendafilova et al., 2012).

Let $x_{i}(l)$ and $x_{j}(k)$ be two signals measured on a vibrating system. The mutual information function between $x_{i}$ and $x_{j}$ is defined as:

$M_{x_{i}, x_{j}}(n, m)=I\left(x_{i}(n), x_{j}(n+m)\right)$

The right hand side of (5) is the mutual information between the signals $x_{i}(l)$ and $x_{j}(k)$ which can be expressed in terms of their probability densities as:

$$
I\left(x_{i}, x_{j}\right)=\log _{2} \frac{P_{x_{i} x_{j}}\left(x_{i}(l), x_{j}(k)\right)}{P_{x_{i}}\left(x_{i}(l)\right) P_{x_{j}}\left(x_{j}(k)\right)}
$$


where $P_{x_{i} x_{j}}$ is the joint probability density function of the signals $x_{i}$ and $x_{j}$ and $P_{x_{i}}$ and $P_{x_{j}}$ are the individual probability densities of $x_{i}(l)$ and $x_{j}(k)$ respectively.

The mutual information measures the full dependence between two signals $x_{i}$ and $x_{j}$, while the cross-correlation only measures linear dependence (the linear part). It can be shown for instance that if the signals $x_{i}$ and $x_{j}$ are linearly related $\left(x_{j}\right.$ is a shifted and attenuated/amplified version of $x_{i}$ ) then both, their cross correlation and their mutual information, will be nonzero (for the shift between the two signals) (Roshni et al., 2008; Trendafilova et al., 2012). But if the relation between two signals is purely nonlinear (e.g. $\left.x_{i}=\left|x_{j}\right|\right)$ then it can be shown that their cross-correlation is zero for all $m$, while their mutual information is nonzero (Roshni et al., 2008). The average over all measurements of the mutual information statistic, the average mutual information (AMI) between $x_{i}$ and $x_{j}$ is

$$
I_{x_{i} x_{j}}=\sum_{x_{i}, x_{j}} P_{x_{i} x_{j}}\left(x_{i}, x_{j}\right) \cdot \log _{2} \frac{P_{x_{i} x_{j}}\left(x_{i}, x_{j}\right)}{P_{x_{i}}\left(x_{i}\right) P_{x_{j}}\left(x_{j}\right)}
$$

It measures the average amount of information learned between the two signals. The AMI varies between 0 and 1 . It will be 0 if two signals are completely independent so that:

$$
P_{x_{i} x_{j}}\left(x_{i}, x_{j}\right)=P_{x_{i}}\left(x_{i}\right) \cdot P_{x_{j}}\left(x_{j}\right) .
$$

On most occasions the relation between the two signals $x_{i}$ and $x_{j}$ is not known, and thus the AMI should be estimated from observations of the signals $x_{i}$ and $x_{j}$. It is known from information theory that the mutual information between two signals is the limit of the mutual information between their quantized versions (Roshni et al., 2008). Thus the average mutual information can be estimated from observations by partitioning the signals $x_{i}$ and $x_{j}$ into nonintersecting intervals. The estimate of the mutual information is simply calculated as a finite sum over all the cells of the partition. In this study the algorithm from (Roshni et al., 2008) is used to obtain an estimate of the average mutual information between two signals. 
Similarly to the cross-correlation it can be shown that for the case of white noise random excitation the mutual information between two signals measured on a vibrating structure does not depend on the excitation signal (Yang et al., 2009; Wang et al., 2010). It only depends on the structural characteristics like mass, stiffness, damping and modal parameters. Thus it can be argued that similarly to the cross correlation the mutual information will also change as a result of damage. As was previously mentioned the cross correlation will detect the dependence between two signals measured on a linearly vibrating structure. But for the case of a nonlinearly vibratory behaviour the two signals $x_{i}$ and $x_{j}$ will not be linearly related and thus the cross-correlation will not be able to detect their dependence as well as the change in it. While the AMI being a measure for full dependence will detect the relation between the two signals as well as changes in this dependence. Accordingly this study suggests the AMI as a nonlinear analogue to the cross correlation which is appropriate to use especially for cases of nonlinear vibratory behaviour. The average mutual information is used like cross correlation for image comparison (Roshni et al., 2008). In this study the average mutual information $I_{x y}$ (see equation (7)) similarly to the cross correlation is suggested as a damage metric.

\section{Simulated example: 2 DOF spring-mass-damper nonlinear system.}

This simple example is used in order to check the performance of the mutual information damage metric as compared to the cross- correlation one and their sensitivity to damage, which is simulated by stiffness, decrease.

\subsection{The system.}

A two-degrees of freedom nonlinear spring-mass-damper system is considered. The nonlinearity is introduced by a quadratic stiffness. Consider the two degrees of freedom system shown which is described by the following equation (see Figure 1): 
$[M] \ddot{x}+[C] \dot{x}+[K] x+f(x)=F$

where: $M=\left[\begin{array}{cc}m_{1} & 0 \\ 0 & m_{2}\end{array}\right] ; \quad C=\left[\begin{array}{cc}c_{1}+c_{2} & -c_{2} \\ -c_{2} & c_{2}\end{array}\right] ; \quad K=\left[\begin{array}{cc}k_{1}+k_{2} & -k_{2} \\ -k_{2} & k_{2}\end{array}\right] ; \quad F=\left[\begin{array}{c}0 \\ F(t)\end{array}\right]$

In this case the nonlinearity is introduced by quadratic restoring force between the two masses:

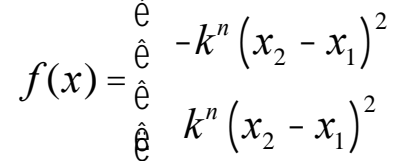

A Gaussian random force $F(t)$ is applied at the second mass $m_{2}$. The displacements of the masses $\mathrm{m}_{1}$ and $\mathrm{m}_{2}, x_{1}(t)$ and $x_{2}(t)$ are used as the two signals measured on the structure.

\subsection{Damage detection.}

As a first example linear stiffness $\mathrm{k}_{2}$ has been changed by reducing it in three different stages vis. with $10 \%, 20 \%$ and $30 \%$ to simulate small, medium and large damage. The results for the maximum cross correlation and for the AMI are shown in Table 1 and in Figure 2. It can be observed that in this case both metrics are able to detect the change in the linear stiffness and to track this change. Both metrics change significantly enough for the lowest level of stiffness reduction and then continue to change for the subsequent damage levels.

As a second example the nonlinear stiffness $\mathrm{k}_{\mathrm{n}}$ has been varied by the same percentages as in the previous example, that is by $10 \%, 20 \%$ and $30 \%$. The results are presented in Table 2 and in Figure 3. One can see that in this case the cross-correlation is not able to detect this damagethe changes are very small and can be neglected. Since this is a change in the nonlinear stiffness it is suggested to be responsible for the nonlinear part of the relation between the two signals measured at $\mathrm{m}_{1}$ and $\mathrm{m}_{2}$. As was mentioned earlier cross-correlation does not detect nonlinear dependence and hence its smaller value for the initial undamaged system and its insensitivity to changes in the nonlinear stiffness. While the AMI performs rather well with nonlinear stiffness changes, very much the same way as with linear stiffness decrease. It detects $10 \%$ the stiffness 
change and then continues changing with the stiffness decrease showing detectable changes for each new damage level.

Thus this example of a two-degree of freedom system shows that both the crosscorrelation and the mutual information can be used to detect and track linear stiffness changes but for changes in the nonlinear stiffness the mutual information is a better choice to detect as well as to track it.

\section{Experiment with a composite beam.}

In this case study the application of the two damage metrics for delamination detection and localization in a composite beam is presented. Composite materials are inherently nonlinear and are known to exhibit well-expresses nonlinear dynamic/vibratory behavior which motivates the choice of the tested structures. (Sanders et al., 1992; Iwasaki et al., 2004).

$1 \mathrm{~m}$ long and $0.06 \mathrm{~m}$ wide woven carbon fibre/epoxy matrix composite beams made of ten layers are used. The beams are clamped at both sides (see Figure 4). One second long broadband random signals sampled at $1000 \mathrm{~Hz}$, which cover the first several modes of the beams, are used as excitation. The maximum amplitude of the signals is kept restricted from above in order to not introduce additional damage to the specimens and from below - to drive them into nonlinear regime. The beams are subjected to force excitation and the accelerations in 10 points, as shown in Figure 4, are measured by piezoelectric accelerometers. Each experiment is conducted 20 times and then the averages of all the 20 realizations used. The standard deviations do not exceed $3 \%$. Thus in this particular case we are using 10 acceleration signals $x_{1}, x_{2}, \ldots x_{10}$ to determine the mutual information and the cross correlation characteristics. These characteristics are determined for a couple of signals $x_{i}$ and $x_{j}$. It can be seen from Figure 4 that one of the measurement points $x_{1}$ is at bottom of the beam, the other 9 measurement points $x_{j}, j=1,2,3, \ldots, 9$ are on the top of the beam. The point $x_{1}$ is at a distance $\mathrm{L} / 10$ from the left end of the beam and the points $x_{j}$ are 
equidistantly distributed over the upper surface of the beam. Thus we calculate 9 cross correlations and 9 AMI's corresponding to the signal couples $\left(x_{1}, x_{j}\right)$, where $j=1,2,3 \ldots, 9$. This is done for the purposes of delamination localization. It is perceived that the closer the measurement point to the delamination the bigger the changes in the measured signal (as compared to the nondelaminated one). And this will result in bigger changes in the dependence/correlation between $x_{1}$ and the signal $x_{j}$, closest to the delamination.

The capabilities of the AMI and the maximum cross correlation to detect delamination are tested experimentally using different delamination sizes and locations. Delamination is introduced between two layers in three different positions along the beam thickness, vis. between the upper two layers (Upper) between the layers 9 and 10 (Lower) and in the middle between layers 5 and 6 (Middle) and in three different positions along the length of the beam, vis. $100 \mathrm{~mm}$ from the left end (Left), in the middle (Centre) and $100 \mathrm{~mm}$ from the right end (Right) (see Figure 5). Delamination is introduced over the whole width of the beam and has different lengths: $0.01 \mathrm{~m}$ (small), $0.02 \mathrm{~m}$ (medium) and $0.03 \mathrm{~m}$ (large).

10 different specimens have been tested: one without delamination and nine others with each type of delamination with respect to the location along the length and the thickness of the beam (Figure 5). Specimens are manufactured as follows:

- $\quad$ one non-delaminated (ND) specimen;

- three specimens with delamination in the Left end side of the specimen: in the upper part, between layers 1-2 (UL), in the middle, between layer 5-6 (ML), and lower, between layers 9-10) (LL);

- three specimens with delamination in the Centre along the specimen length: in the upper (UC) the middle (MC), and lower part (LC) along the specimen thickness;

- three specimens with delamination in the Right end side of the specimen: in the upper (UR), in the middle (MR) and in the lower (LR) part. 
Initially beams with small delamination are tested, and then the delamination size is increased first to medium (20mm long) and eventually to long (30 mm long).

Two damage indexes based on the two above mentioned metrics are introduced, which give their relative percentage changes of the two metrics- the cross-correlation and the AMI. The $\omega_{1 j}$ represents the relative percentage change in the cross-correlation as compared to the nodelamination case for the points $\mathrm{x}_{1}$ and $x_{j}, j=1,2, \ldots 9$ :

$$
\omega_{x_{1}, x_{j}}=\frac{\left(\Omega_{x_{1} x_{j}}^{i n}-\Omega_{x_{1} x_{j}}^{\text {dam }}\right)}{\Omega_{x_{1} x_{j}}^{i n}} .100
$$

where $\Omega_{{ }_{1} x_{j}}^{i n}$ is the cross-correlation corresponding to the initial state, which is assumed undamaged and $\Omega_{x_{1} x_{j}}^{d a m}$ is the cross-correlation corresponding to the current possibly damaged state. In a similar way a delamination index based on the AMI is now introduced, which represents the relative percentage change in the AMI between the baseline (undamaged) condition and the current possibly damaged one for two signals $x_{1}$ and $x_{j}$, where $j=1,2 . ., 9$ :

$i_{x_{1} x_{j}}=\frac{I_{x_{1} x_{j}}^{i n}-I_{x_{1} x_{j}}^{\text {dam }}}{I_{x_{1} x_{j}}^{i n}} .100$

\section{$5 \quad$ Results obtained using both features.}

In this paragraph some results obtained for the damage scenarios presented in $\& 4$ are introduced. We shall first refer to the results for the cross-correlation and the mutual information between the measured signals. The normalized cross-correlations as given by equation (3) are in the range of 0.5, which is much smaller than 1 for both the delaminated and the non-delaminated specimens, while the average mutual information is 0.9 for the non-delaminated specimens going down to about 0.75 for the delaminated ones (see Tables 3a) and 3b)). Thus it can be concluded that the 
initial dependence of the signals (for the case of an undamaged structure) in terms of cross correlation is somewhat low, which suggests that the linear dependence between two signals is low. On the other hand the values of the mutual information are quite high for the case of the undamaged structure which implies the presence of nonlinear dependence between two signals measured on the structure. Both characteristics do go down as a result of the introduction and the growth of delamination, but the change in the mutual information is much more prominent as compared to the change in the cross correlation. This again can be considered as an implication that delamination itself changes mostly the nonlinear dependence between two signals measured on a structure. It does affect their linear correlation as well but this change is much smaller as compared to the change in the AMI.

\subsection{Delamination detection.}

Our first aim is to check the ability of the two damage indexes to detect delamination in the composite beam tested. The performance of the cross correlation-based index $\omega_{1 j}$ introduced by equation (10) are firstly checked. The maximum value of $\omega_{1 j}$ for the signals $x_{j}, j=1,2, . .9$, is used for delamination detection.

$$
\omega_{x_{1} x_{j}}=\max _{j} \omega_{x_{1} x_{j}}
$$

Table 3a) gives the results. It can be seen that the cross-correlation based index is not very helpful in detecting small delamination in the composite beam tested. It varies between $2.5 \%$ and $5.3 \%$ for the different delamination locations. It exceeds $5 \%$ only for the cases of small delamination, vis the middle and the lower case of delamination in the right hand side of the beam. The changes are bigger for the cases of medium and large delamination and these should be correctly detectable using the cross-correlation-based index defined in (10). In a similar way the performance of the AMI-based index defined in (11) are studied. Again the maximum value for all the measurement points is used for delamination detection purposes: 
$i_{x_{1} x_{j}}=\max _{j} i_{x_{1} x_{j}}$

Table $3 \mathrm{~b}$ represents the results for the AMI-based index $i_{x_{1} x_{j}}$. It can be seen that it is much more sensitive to delamination as compared to the cross-correlation. It changes from $5.39 \%$ to about $8.13 \%$ for the case of small delamination. The index increases for medium and large delamination cases.

\subsection{Delamination localization.}

The signals $x_{j}$ are measured in 9 different points, $j=1,2, . ., 9$. These signals will be used for delamination localization purposes. As was mentioned above it was found that the two indexes $\omega_{1 j}$ and $i_{x_{1} x_{2}}^{j}$. are sensitive to the damage location and the closer the point to the delamination the more the indexes are affected. This is the reason to vary the location of one of the measurement points. As a matter of fact all nine cross correlation indexes $\omega_{x y}^{j}$ and $i_{x_{1} x_{2}}^{j}$ corresponding to the nine locations on the beam change. But the biggest changes are found when the measurement point $x_{j}$ is close to the delamination location.

It is shown that the cross-correlation index cannot detect small delamination and thus the results for the index $\omega_{1 j}$ are for the case of medium delamination. They are presented in Figure 6. In a similar way the AMI-based index $i_{x_{1} x_{j}}$ for small delamination is presented for the 9 measurement points along the beam in Figure 7. It can be seen that the AMI-based indexes locate the delamination quite clearly - the maximum index corresponds to the location of the delamination. This can be claimed for the cross-correlation-based index $\omega_{1 j}$ to a certain extent only. It has its maximum value for the location of the delamination, but in some cases it has relatively high values for other locations as well, which might be misleading. 


\section{Discussion.}

This work discusses the application of the mutual information between two signals measured on a vibrating structure subjected to unknown random excitation for the purposes of delamination diagnosis. The mutual information is regarded as a nonlinear analogue to the linear signal cross correlation and from such a perspective its performance is compared to that of the cross correlation. Delamination detection and localization indexes are introduced based on the signal cross-correlation and on the average mutual information between two signals. The performance of the cross correlation and the AMI are first compared for the case of a simulated example for the purposes of linear and nonlinear stiffness reduction. It is shown that while for the case of linear stiffness change both metrics are capable to detect the changes, for the case of nonlinear stiffness reduction the mutual information presents a far better alternative- the cross correlation fails to detect these changes unanimously.

The method is then demonstrated for the purposes of delamination diagnosis in composite laminate beams. In this particular case the suggested characteristics demonstrate quite good capabilities for delamination detection and localization with experimentally measured signals. Regarding the application of the method and the suggested characteristics for different materials and other types of composites or structures, the developed method can be applied for other types of structures and materials since the methodology is based on relatively simple signal characteristics, expected to change as a result of the presence of a damage. But it should be noted that for each particular case a proper calibration is needed to fit the method for the particular structure and/or material. This requires a proper experimental campaign in order to establish the sensitivity of the suggested characteristics with the change of damage/delamination and its location.

More care and attention is needed regarding the localization of damage/delamination and certainly the suggested method cannot be automatically applied for different structures and 
materials. It should be noted that this application attempts to perform crude localization, which is to identify the area where the damage/delamination is most likely to be. And in such a sense the method should be applicable for other types of materials and structures. The approach suggested is attractive from a practical view point since it only requires two time domain signals measured in different points on a vibrating structure subjected to unknown random excitation to detect delamination. The suggested indexes can be calculated in a rather straightforward manner from the measured signals. Thus this method is easy to apply in practice and it was shown to predict with good precision the presence and the location of delamination in composite laminates. The suggested AMI-based metric and index are primarily aimed for structures with nonlinear vibratory behaviour such as those made of composites. But it was shown to be general enough and it should be capable to diagnose damage in linearly vibrating structures. The simplicity of the method and its generality make it appropriate for on-line, real-time monitoring of different structures, such as airplane wings. But again a proper testing and calibration is needed to fit the method for a particular structure and/or material.

The suggested AMI-based index demonstrates sensitivity to the delamination extent, which suggests its capability for delamination quantification. Thus one natural development of the method will be focused on catching the depth of the delamination, which is the last step for a complete delamination diagnosis in composite laminate structures. 


\section{REFERENCES (first author's alphabetically ordered)}

Bendat, J.S., Piersol, A., 2011. Random data: Analysis and measurement Procedures, IV edition, J. Wiley.

Cawley, P. and Adams, R.D., 1979. The locations of defects in structures from measurements of natural frequencies. Journal of Strain Analysis 14, 49-57.

Diaz Valdes, S.H., Soutis, C., 1999. Delamination detection in composite laminates from variations of their modal characteristics. Journal of Sound and Vibration 228, 1-9.

Doebling, S.W., Farrar, C.R., Prime, M.B., 1998. A summary review of vibration-based damage identification methods. The Shock and Vibration Digest 30, 91-105

Iwasaki, A., Todoroki, A., Shimamura, Y., Kobayashi, H., 2004. Unsupervised structural damage diagnosis based on change of response surface using statistical tool (Application to damage detection of composite structure). JSME International Journal, Series A: Solid Mechanics and Material Engineering 47, 1-7

Kantz, H., Schreiber, T., 2004. Nonlinear time series analysis, II edition, Cambridge University Press.

Le Wang, Zhichun Yang, T.P. Waters, Structural damage detection using cross correlation functions of vibration response. Journal of Sound and Vibration 329, 5070-5086.

Minak, G., Palazzetti, R., Trendafilova, I., Zucchelli, A., 2010. Delamination localization and length estimation in composite laminate beam by VSHM and pattern recognition methods. Mechanics of Composite Materials, 46, 387-394.

Nichols, J.M., 2006. Examining structural dynamics using information flow. Probabilistic Engineering Mechanics 21, 420-433. 
Nichols, J.M., Seaver, M., Trickey, S.T., 2009. A method for detecting damage-induced nonlinearities in structures using information theory. Journal of Sound and Vibration 322, 438453.

Overbey, L.A., Todd, M.D., 2009. Dynamic system change detection using a modification of the transfer entropy. Journal of Sound and Vibration 322, 438-453

Roshni, V.S., Revathy, K., 2008. Using mutual information and cross correlation as metrics for registration of images. Journal of Theoretical and Applied technology 4, 474-481.

Sanders, D., Kim, Y.I., Stubbs, R.N., 1992. Nondestructive evaluation of damage in composite structures using modal parameters. Experimental Mechanics 32, 240-25.

Trendafilova, I., 2006. Vibration-based damage detection in structures using time series analysis. Journal of Mechanical Engineering Science, Proceedings of the Institution of Mechanical Engineers Part C 220, 261-272.

Trendafilova, I., Heylen, W., Van Brussel, H., 2001. Measurement point selection in damage detection using the mutual information concept. Smart Materials and Structures 10, 528-533. Trendafilova, I., Palazzetti, R., Zucchelli, A., 2012. Delamination assessment in structures made of composites based on signal cross-correlation, Proceedings ISMA2012, Leuven, Belgium Trendafilova, I., Manoach, E., 2008. Vibration based damage detection in plates by using time series analysis. Mechanical Systems and Signal Processing 22, 1092-1106.

Yang, Z., Wang, L., Wang, H., Ding, Y., Dang, X., 2009. Damage detection in composite structures using vibration response under stochastic excitation. Journal of Sound and Vibration $325,755-768$.

Yang, Z., Yu, Z., Sun, H., 2007. On the cross correlation function amplitude vector and its application to structural damage detection. Mechanical Systems and Signal Processing 21, 29182932. 
Yu, Z., Yang, Z., 2007. Damage detection based on the cross correlation function amplitude vector and its application to the ASCE benchmark structure. Key Engineering Materials 353-358, 2317-2320. 


\section{FIGURE CAPTIONS}

FIGURE 1: The 2 DOF system

FIGURE 2: 2 DOF system cross-correlation and AMI changes with linear stiffness decrease

FIGURE 3: 2 DOF system cross-correlation and AMI changes with nonlinear stiffness decrease

FIGURE 4: The composite beam tested and the measurement points

FIGURE 5: Delamination positions and sizes: a) horizontal position, b) vertical position and c) sizes

FIGURE 6: Cross-correlation-based index for a) left-hand delamination, b) central delamination and c) right-hand delamination

FIGURE 7: AMI-based index for a) left-hand delamination, b) central delamination and c) righthand delamination 


\section{TABLE CAPTIONS}

TABLE 1: Percentage changes of the cross-correlation and the AMI for 2-DOF system with linear stiffness decrease

TABLE 2: Percentage changes of the cross-correlation and the AMI for 2-DOF system with nonlinear stiffness decrease

TABLE 3a: Cross correlation values and indexes with delamination

TABLE 3b: AMI values and indexes with delamination

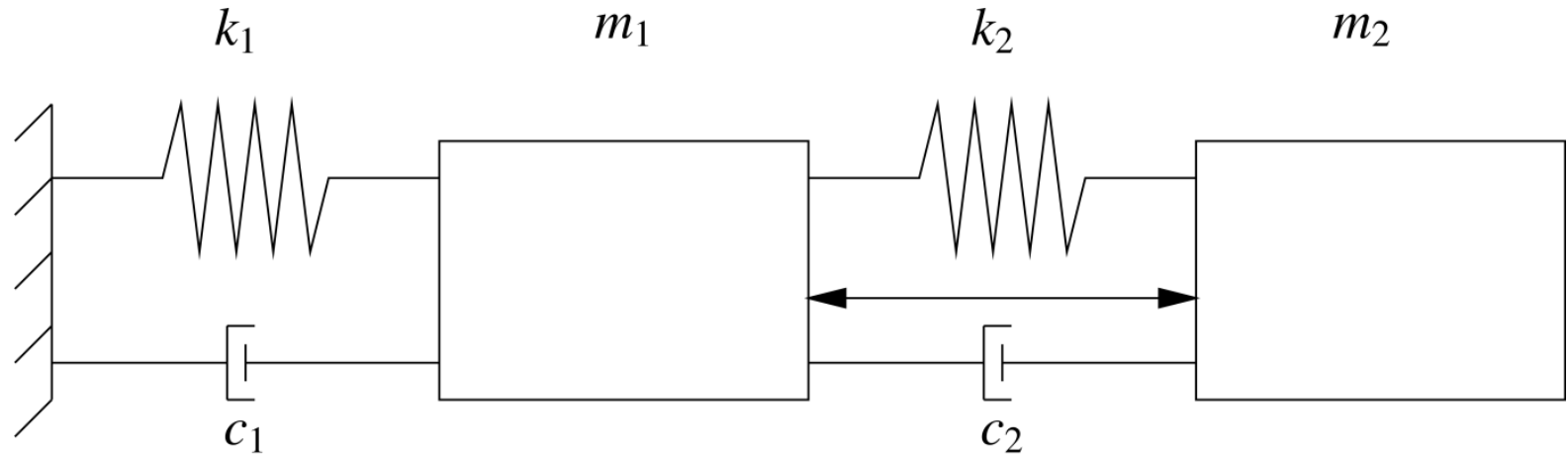

Figure 1 


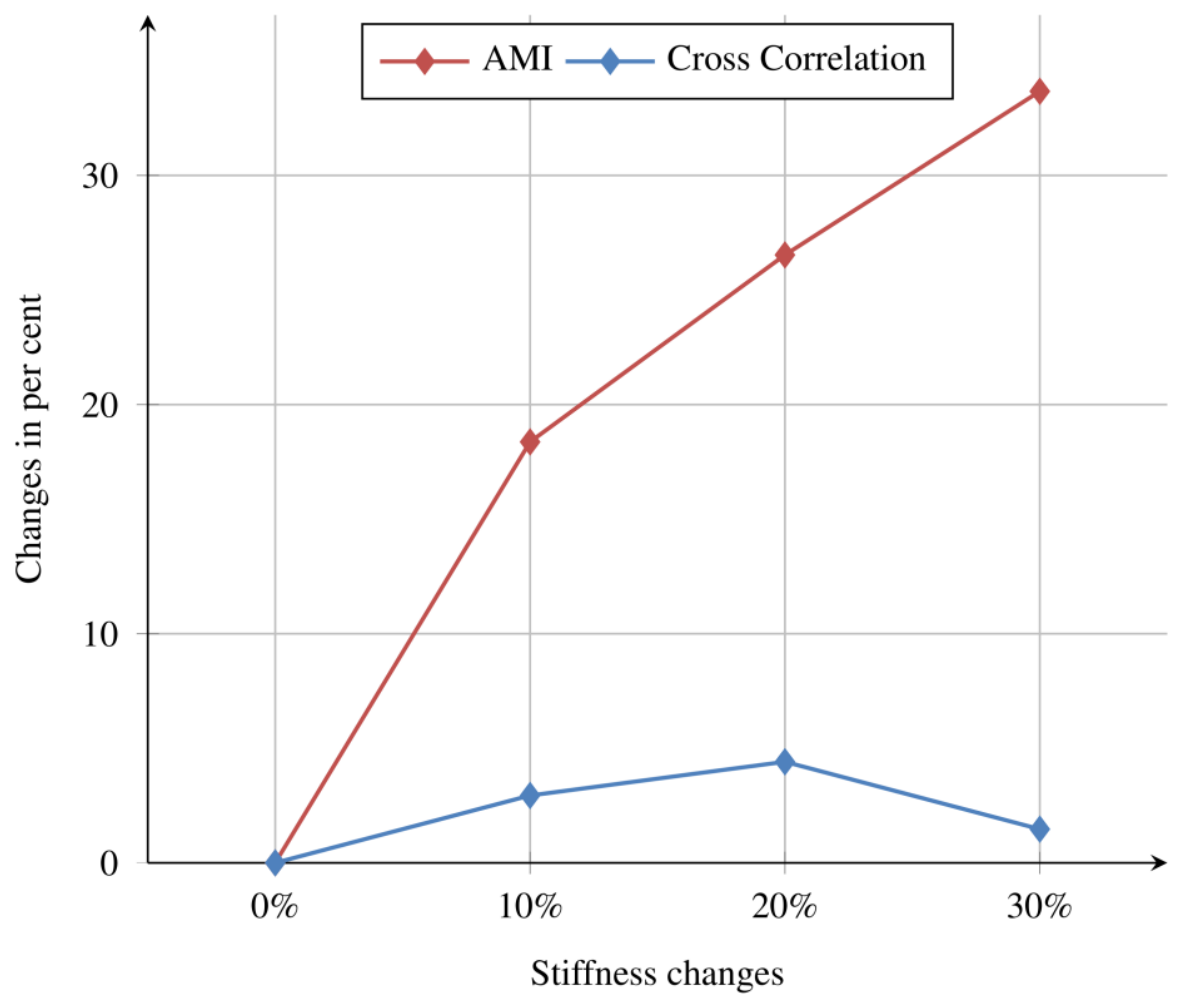

Figure 2 


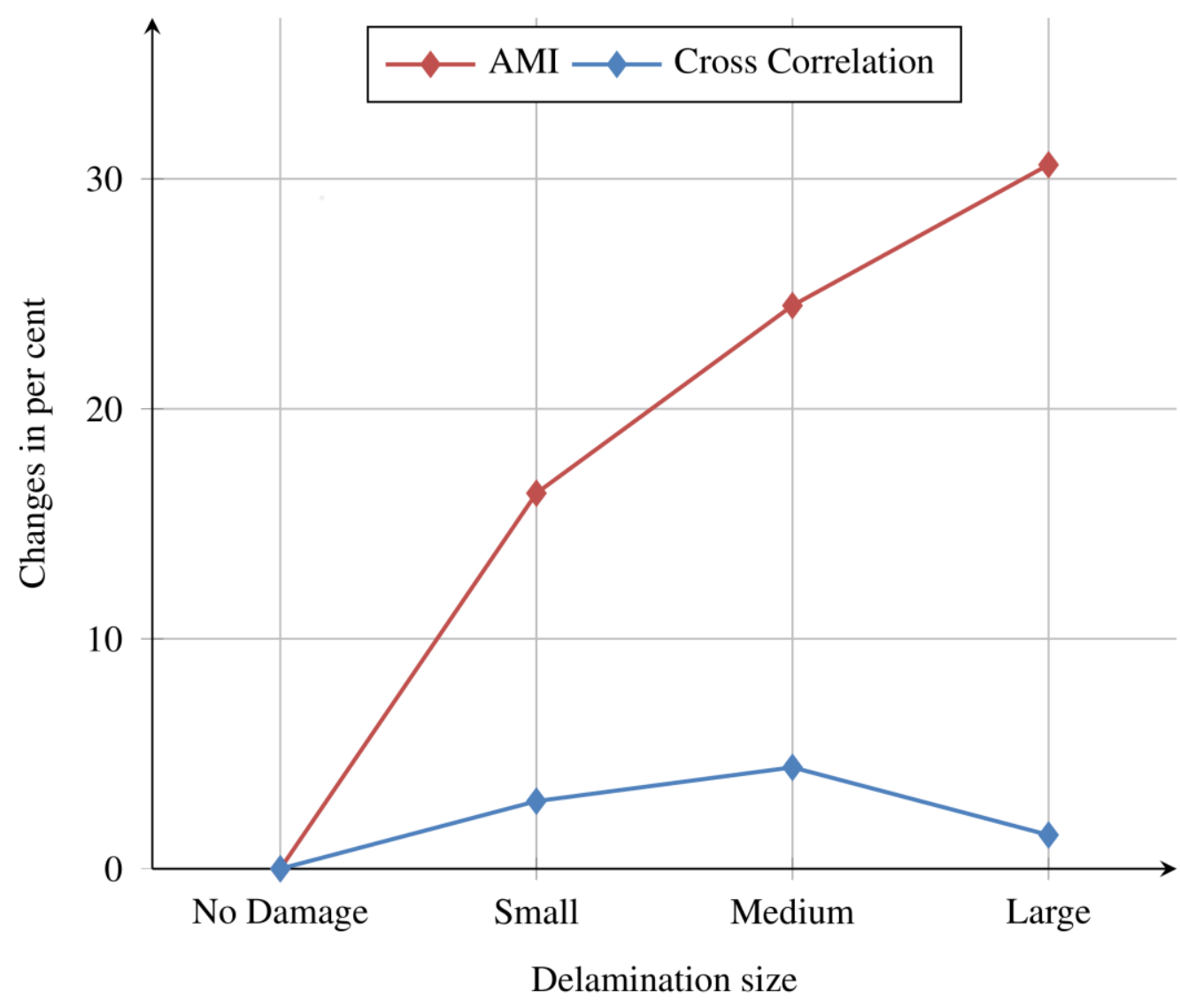

Figure 3

Signals $x_{j}$ measurement points. $\mathrm{j}=1,2, \ldots, 9$

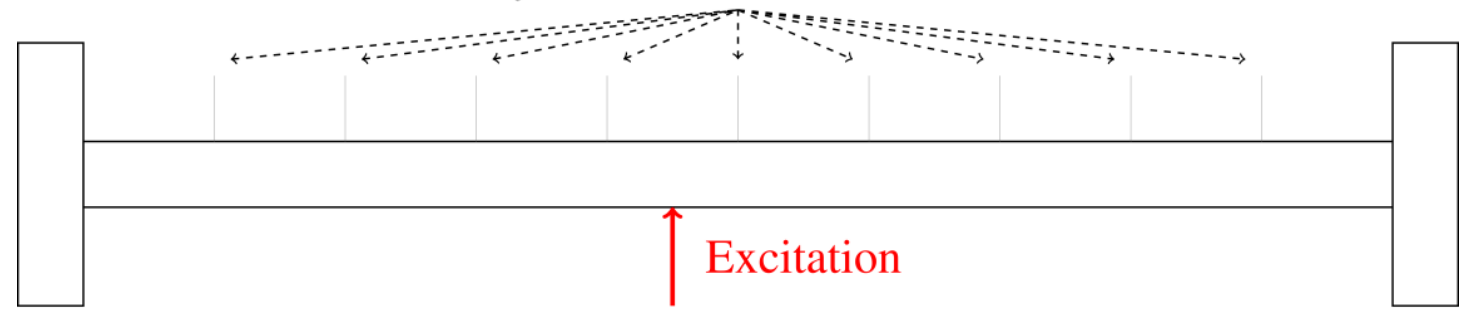

Figure 4 
a)

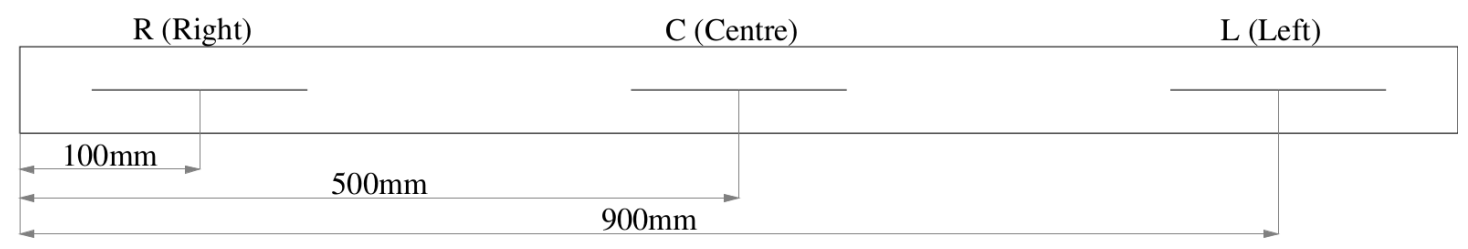

b)

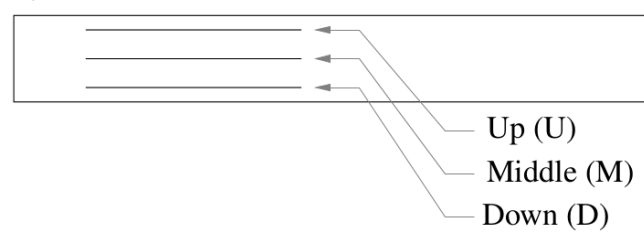

c)

Small 10mm (S)

Medium 20mm (M)

Large $30 \mathrm{~mm}(\mathrm{~L})$

Figure 5
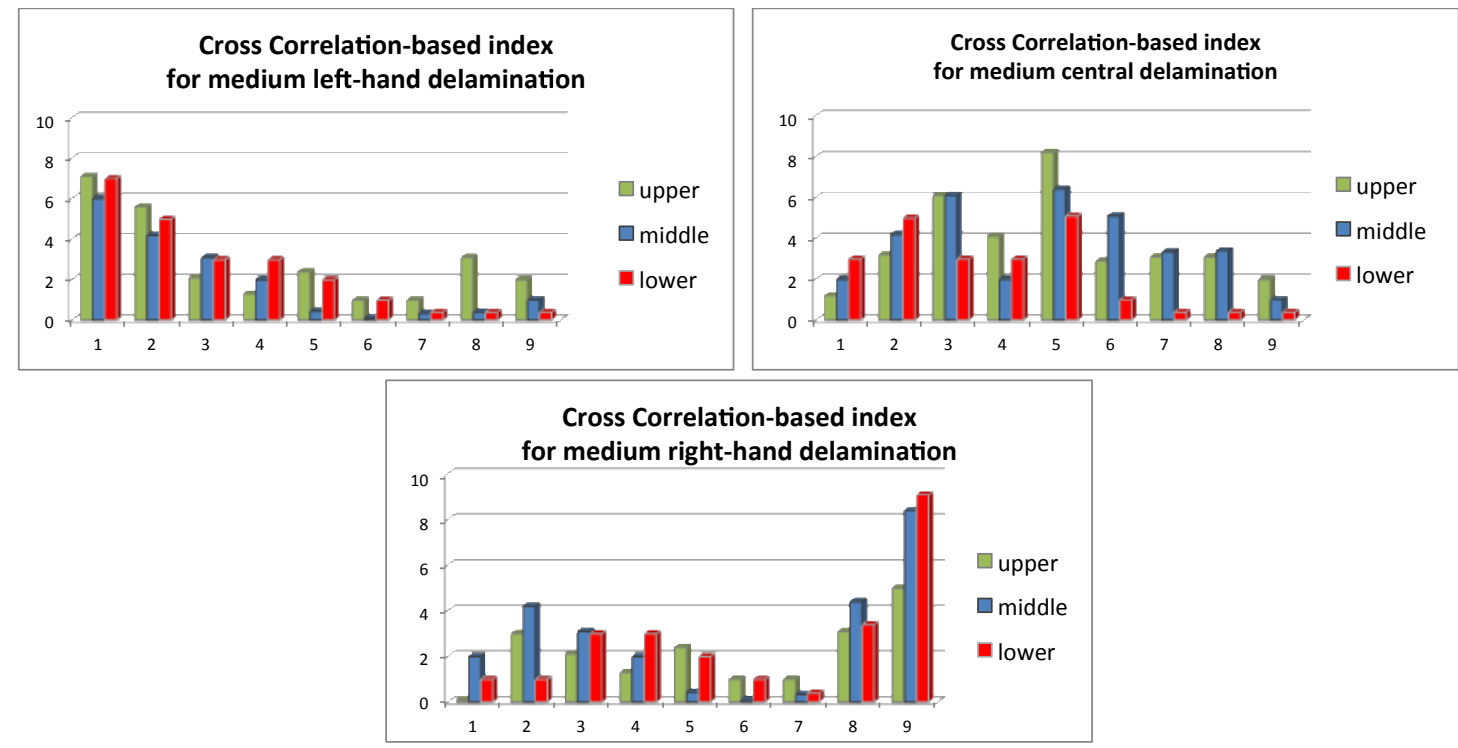

Figure 6 


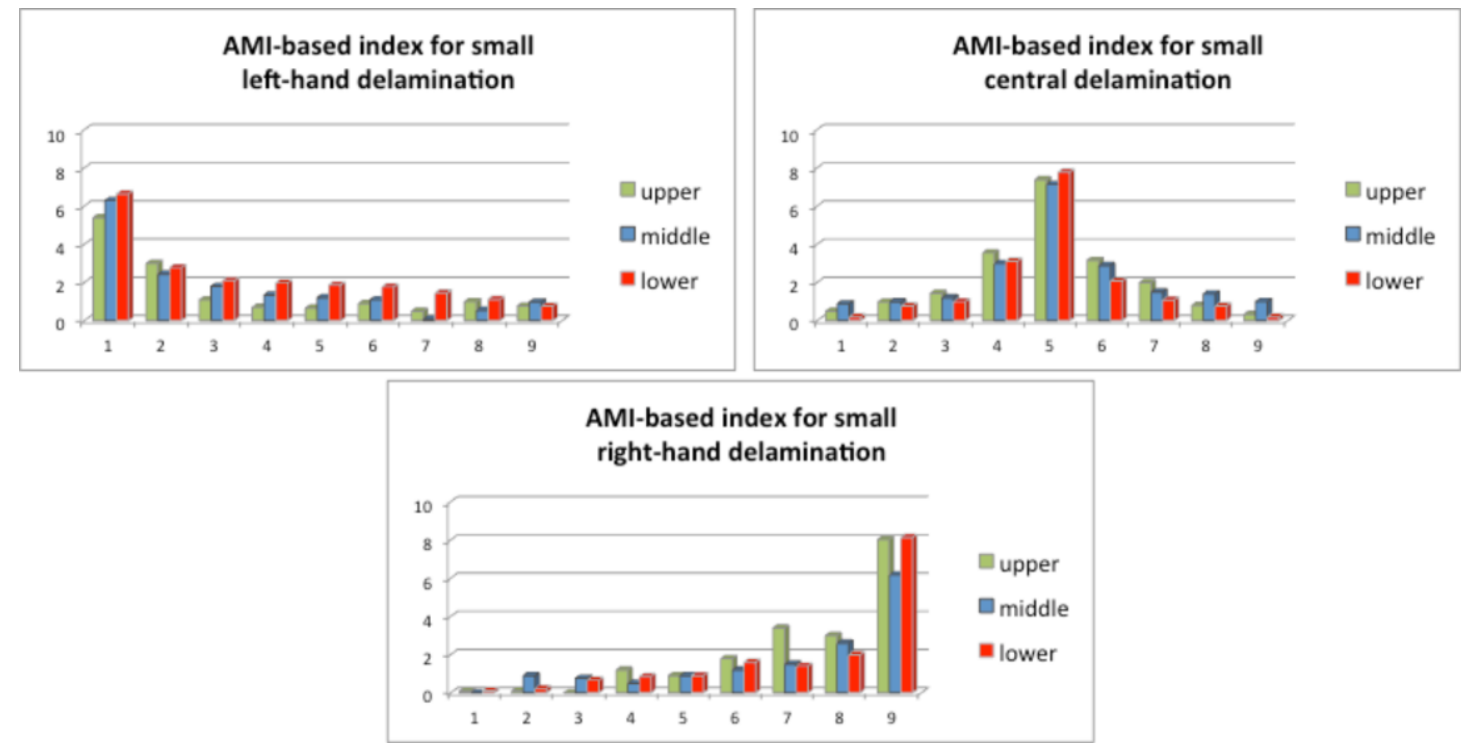

Figure 7

Damage level

No Damage Small Medium Large

Index

0.68

0.59

0.50

0.46

Cross Correlation

\% Change

13.24

19.12

26.47

$\%$ Change from previous level

$5.88 \quad 4.41$

Index

0.98

0.80

0.72

0.65

AMI

\% Change

$18.37 \quad 26.53$

\% Change from previous level

$8.16 \quad 7.14$

Table 1. Percentage changes of the cross-correlation and the AMI for 2-DOF system with linear stiffness decrease 


\section{Damage level}

\begin{tabular}{|c|c|c|c|c|c|}
\hline & & No Damage & Small & Medium & Large \\
\hline \multirow{4}{*}{ Cross Correlation } & Index & 0.68 & 0.66 & 0.65 & 0.67 \\
\hline & \% Change & & 2.94 & 4.41 & 1.47 \\
\hline & $\%$ Change from previous level & & & 1.47 & -2.94 \\
\hline & Index & 0.98 & 0.82 & 0.74 & 0.68 \\
\hline \multirow[t]{2}{*}{ AMI } & $\%$ Change & & 16.33 & 24.49 & 30.61 \\
\hline & $\%$ Change from previous level & & & 8.16 & 6.12 \\
\hline
\end{tabular}

Table 2. Percentage changes of the cross-correlation and the AMI for 2-DOF system with nonlinear stiffness decrease 


\begin{tabular}{|c|c|c|c|c|c|c|c|c|}
\hline \multirow[b]{2}{*}{ No delamination } & \multirow{2}{*}{\multicolumn{2}{|c|}{ Delamination }} & \multicolumn{6}{|c|}{ Delamination size } \\
\hline & & & \multicolumn{2}{|c|}{ Small } & \multicolumn{2}{|c|}{ Medium } & \multicolumn{2}{|c|}{ Large } \\
\hline \multirow[t]{2}{*}{$\omega_{\mathrm{xy}}$} & & & $\omega_{\mathrm{xy}}$ & Change & $\omega_{\mathrm{xy}}$ & Change & $\omega_{x y}$ & Change \\
\hline & & Upper & 0.531 & $3.40 \%$ & 0.511 & $6.02 \%$ & 0.494 & $10.11 \%$ \\
\hline \multirow[t]{3}{*}{0.55} & Left & Middle & 0.536 & $2.55 \%$ & 0.517 & $7.00 \%$ & 0.484 & $12.00 \%$ \\
\hline & & Lower & 0.531 & $3.40 \%$ & 0.512 & $8.23 \%$ & 0.488 & $11.21 \%$ \\
\hline & & Upper & 0.534 & $3.00 \%$ & 0.505 & $8.23 \%$ & 0.499 & $9.34 \%$ \\
\hline \multirow[t]{3}{*}{0.55} & Centre & Middle & 0.525 & $4.60 \%$ & 0.515 & $6.41 \%$ & 0.49 & $10.44 \%$ \\
\hline & & Lower & 0.529 & $3.90 \%$ & 0.522 & $5.10 \%$ & 0.493 & $10.12 \%$ \\
\hline & & Upper & 0.53 & $3.60 \%$ & 0.523 & $5.00 \%$ & 0.494 & $11.67 \%$ \\
\hline \multirow[t]{2}{*}{0.55} & Right & Middle & 0.521 & $5.30 \%$ & 0.504 & $8.40 \%$ & 0.486 & $12.01 \%$ \\
\hline & & Lower & 0.521 & $5.20 \%$ & 0.5 & $9.09 \%$ & 0.484 & $10.44 \%$ \\
\hline
\end{tabular}

Table 3a. Cross correlation-based index $\omega_{x y}$ with delamination 


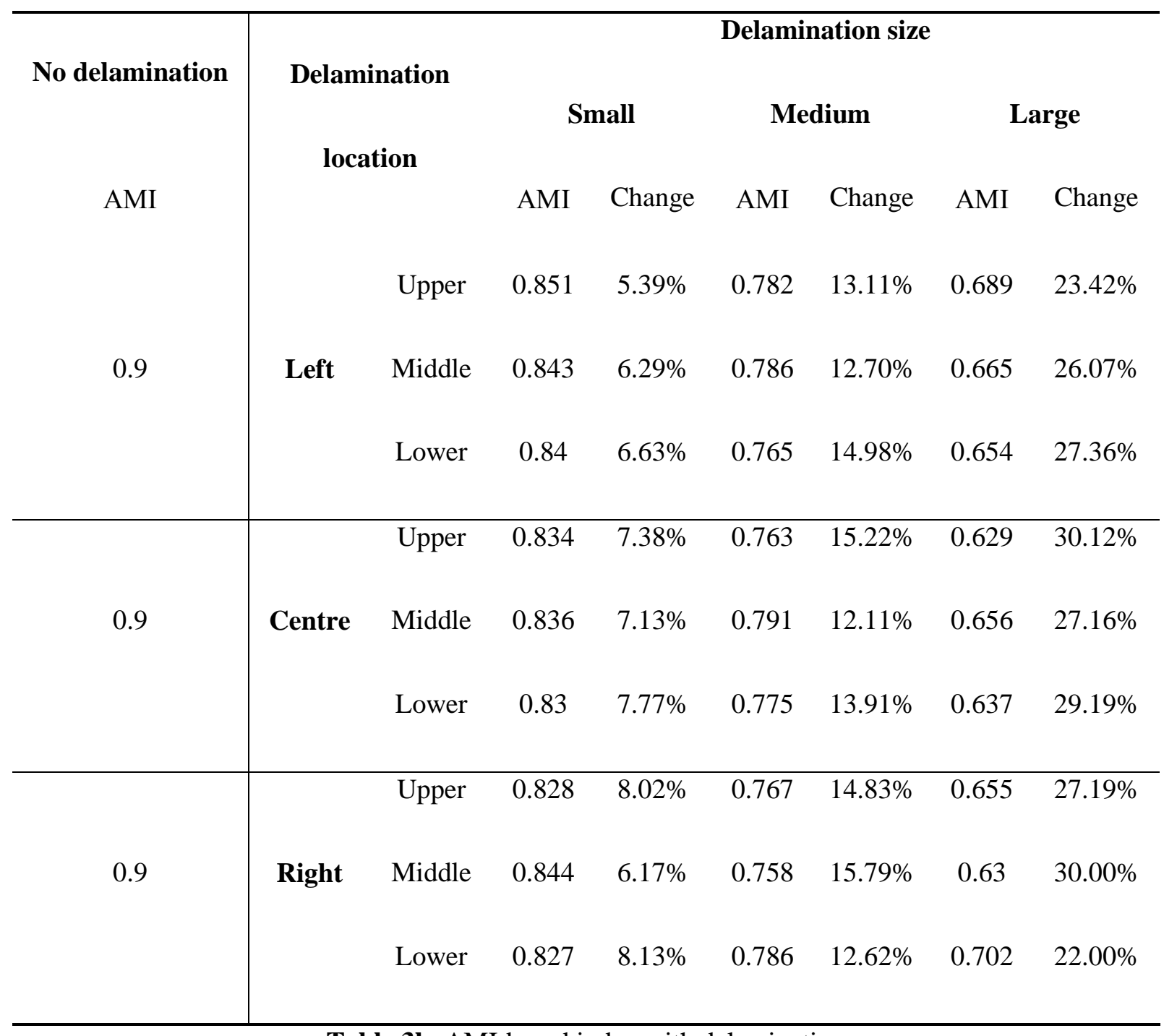

Table 3b. AMI-based index with delamination 
